\title{
A tuberculose na população em situação de rua: representações sociais de profissionais da Atenção Primária à Saúde
}

\author{
Tuberculosis in the homeless population: social representations of Primary Health Care \\ professionals
}

Tuberculosis en la población sin hogar: representaciones sociales de los profesionales de la Atención Primaria de Salud

\author{
Flávia Thayanne Barbosa de Sousa \\ ORCID: https://orcid.org/0000-0002-9583-2648 \\ Universidade Federal de Campina Grande, Brasil \\ E-mail: flaviathayanne@ hotmail.com \\ Rodrigo Pinheiro Fernandes de Queiroga \\ ORCID: https://orcid.org/0000-0002-0642-6382 \\ Universidade Federal de Campina Grande, Brasil \\ E-mail: rodrigopfq@gmail.com \\ Daniele Mamédio de Andrade \\ ORCID: https://orcid.org/0000-0002-3291-1525 \\ Universidade Federal de Campina Grande, Brasil \\ E-mail: danieleandrade300791@gmail.com \\ Sheila Milena Pessoa dos Santos \\ ORCID: https://orcid.org/0000-0001-9396-9192 \\ Universidade Federal de Campina Grande, Brasil \\ E-mail: sheila.milena@gmail.com \\ Sthephanie de Abreu Freitas \\ ORCID: https://orcid.org/0000-0001-8219-5013 \\ Universidade Federal da Paraíba, Brasil \\ E-mail: stheabreu@hotmail.com
}

\begin{abstract}
Resumo
Objetivo: Compreender as representações sociais de profissionais de saúde sobre a atenção à Tuberculose (TB) na População em Situação de Rua (PSR). Método: Estudo exploratório, descritivo, de abordagem qualitativa realizado em Campina Grande - PB. A população foi constituída por profissionais de ensino superior da Atenção Primária à Saúde (APS); 45 profissionais aceitaram participar da pesquisa. Para a coleta de dados, realizada entre abril e julho de 2017, foram realizadas entrevistas semiestruturadas e preenchimento de questionários. As entrevistas foram analisadas com o auxílio do software Iramuteq, através da Classificação Hierárquica Descendente. A análise utilizou o dispositivo teórico-analítico das representações sociais. Resultados: Foram identificadas as seguintes classes: A vida nas ruas: contexto de vulnerabilidade; Desafios para o diagnóstico e tratamento da TB na PSR; Obstáculos para o acesso da PSR à APS; e A problemática da Equipe de Consultório na Rua (ECR). Conclusão: Apesar do reconhecimento do contexto de vulnerabilidade, foi constatada uma série de barreiras ao acesso da PSR à APS. Sobre as ações e organização dos serviços, prevaleceram representações que não consideram as especificidades da PSR. A atuação da ECR mostrou-se seriamente comprometida.
\end{abstract}

Palavras-chave: Controle de doenças transmissíveis; Populações vulneráveis; Acesso aos serviços de saúde.

\begin{abstract}
Objective: To understand the social representations of health professionals about Tuberculosis (TB) care in the Homeless Population (HP). Method: Exploratory and descriptive study, with a qualitative approach, conducted in Campina Grande - PB. The population consisted of higher education professionals from the Primary Health Care (PHC); 45 professionals agreed to take part in the research. For data collection, held between April and July 2017, semi-structured interviews were performed and questionnaires were completed. The interviews were analyzed with the aid of the Iramuteq software, through the Descending Hierarchical Classification. The analysis used the theoretical-analytical device of social representations. Results: The following classes were identified: Life on the streets: context of vulnerability; Challenges for the diagnosis and treatment of TB in HP; Obstacles to HP access to PHC; and The issue of the Street Clinic Team (SCT). Conclusion: Despite the recognition of the context of vulnerability, a series of barriers to HP access to PHC was noted. Concerning the actions and organization of services,
\end{abstract}


representations that do not consider the specificities of HP prevailed. The performance of SCT was seriously undermined.

Keywords: Communicable disease control; Vulnerable populations; Health services accessibility.

\begin{abstract}
Resumen
Objetivo: Comprender las representaciones sociales de los profesionales de la salud sobre la atención de la tuberculosis (TB) en la población sin hogar (PSH). Método: Estudio exploratorio descriptivo con enfoque cualitativo realizado en Campina Grande - PB. La población se compuso de profesionales de educación superior de la Atención Primaria de Salud (APS); 45 profesionales aceptaron participar en la investigación. Para la recolección de datos, realizada entre abril y julio de 2017, se realizaron entrevistas semiestructuradas y se completaron cuestionarios. Las entrevistas se analizaron con la ayuda del software Iramuteq, mediante la Clasificación Jerárquica Descendente. El análisis utilizó el dispositivo teórico-analítico de las representaciones sociales. Resultados: Se identificaron las siguientes clases: La vida en la calle: contexto de vulnerabilidad; Retos para el diagnóstico y tratamiento de la TB en PSH; Barreras para el acceso de la PSH a la APS; y La cuestión del Equipo de Consultorio en la Calle (ECC). Conclusión: A pesar del reconocimiento del contexto de vulnerabilidad, se encontró una serie de barreras para el acceso de la PSH a la APS. En cuanto a las acciones y organización de los servicios, prevalecieron las representaciones que no consideran las especificidades de la PSH. El rendimiento de la ECC se vio seriamente comprometido.
\end{abstract}

Palabras clave: Control de enfermedades transmisibles; Poblaciones vulnerables; Accesibilidad a los servicios de salud.

\title{
1. Introdução
}

A tuberculose (TB) representa um grave problema de saúde pública mundial ao acometer milhares de pessoas anualmente, levando a óbito parcela significativa dessa população. No relatório anual divulgado em 2020, a Organização Mundial de Saúde (OMS) estimou que cerca de 10 milhões de pessoas adoeceram por TB no ano de 2019; dessas, 1,2 milhões de pessoas morreram pela doença. O Brasil continua entre os 30 países de alta carga para a TB, sendo considerado prioritário para o controle da doença no mundo (WHO, 2020).

Alinhado ao esforço global para o enfrentamento da TB, o Brasil elaborou o Plano Nacional de Controle da Tuberculose (PNCT), que se alicerça em três pilares: prevenção e cuidado integrado centrado na pessoa, políticas arrojadas e sistemas de apoio e intensificação da pesquisa e inovação. De acordo com o plano, a rede de serviços em saúde, que envolve as esferas federal, municipal e estadual, deve desenvolver ações que permitam o controle e prevenção, com acesso a medicamentos e ao diagnóstico da doença (Brasil, 2017a).

A TB está associada à pobreza e à iniquidade social. As populações mais carentes, socialmente desamparadas e marginalizadas são atingidas de forma intensa e desproporcional por doenças como a TB, que tem se estabelecido nos países menos favorecidos de forma assustadora (Santos, Vendramini, Gazetta, Oliveira, \& Villa, 2007). Entre os grupos vulneráveis, destaca-se a População em Situação de Rua (PSR), pois suas características e a dinâmica de vivência na rua potencializam a infecção por TB e dificultam o controle da doença. Estimativas do Ministério da Saúde (MS) apontam que, no Brasil, a taxa de incidência da TB na PSR é 56 vezes maior que na população em geral (Brasil, 2019a). A PSR constitui um grupo heterogêneo que não possui moradia regular, tem vínculos familiares rompidos ou fragilizados e desenvolve meios de sobrevivência associados e desenvolvidos na rua (Silva, Viana, Almeida, Santos, \& Nery, 2021).

Vale destacar que a responsabilidade inicial pela atenção à saúde das populações vulneráveis, como de qualquer outro cidadão, é da Atenção Primária à Saúde (APS). Esse nível de atenção à saúde, principalmente através da Estratégia Saúde da Família (ESF), atua como a porta de entrada preferencial do usuário no sistema de saúde, realiza o acompanhamento longitudinal, articula com outros pontos de atenção e regula o fluxo da população aos serviços nos níveis secundários e terciários, atuando, dessa forma, como coordenadora da Rede de Atenção à Saúde - RAS (Brasil, 2017b).

Sobre o atendimento à PSR, susceptível à TB, observa-se que há uma dificuldade dos serviços de saúde, principalmente os pertencentes à esfera da APS, em atender as pessoas que integram a população que vive nessas condições. 
Por encontrar dificuldade de acesso no SUS, muitas pessoas em situação de rua procuram os serviços de saúde apenas quando não suportam mais a presença dos sintomas de doenças e, muitas vezes, acessam o sistema de saúde apenas quando conduzidas pelo serviço de resgate ou por intermédio de instituições de assistência. A principal porta de entrada da PSR no SUS acaba sendo os serviços de urgência e emergência, quando condições crônicas que não foram bem cuidadas culminam em quadros agudos (Hino, Monroe, Takahashi, Souza, Figueiredo, \& Bertolozzi, 2018).

Identificar as dificuldades do serviço de saúde para rastrear, diagnosticar, tratar e acompanhar os casos de TB na PSR implica evidenciar os problemas para melhor atuar no controle da doença.

Nessa compreensão, o estudo pautou-se no seguinte questionamento: Quais as representações sociais dos profissionais de saúde sobre a PSR com TB. Dessa forma, o estudo teve o objetivo de compreender as representações dos profissionais atuantes na APS sobre a atenção à TB na PSR.

O presente estudo justifica-se pela necessidade de produzir conhecimentos teóricos e operacionais acerca dos aspectos que comprometem o acesso e o cuidado às pessoas em situação de rua no âmbito da APS. Espera-se que desvelar como ocorre a prestação da assistência à pessoa em situação de rua com TB possibilite repensar a capacidade dos serviços do SUS, a começar pela APS, em atender as reais necessidades dessa população e no planejamento de políticas de controle da doença, possibilitando estratégias de intervenção que subsidiem o adequado manejo clínico do problema no contexto do Sistema Único de Saúde (SUS), contribuindo, assim, para o incremento na qualidade da assistência prestada a essa população vulnerável.

\section{Metodologia}

Este estudo integra o projeto intitulado: "Análise dos pontos de estrangulamento no controle da tuberculose em populações em situação de rua nos municípios de João Pessoa e Campina Grande - PB”. A pesquisa foi aprovada no Comitê de Ética em Pesquisa (CEP) envolvendo seres humanos do Centro de Ciências da Saúde da Universidade Federal da Paraíba (UFPB), sob o CAAE 20446513.0.0000.5188, atendendo, portanto, às recomendações contidas na Resolução ${ }^{\circ}$ 466/12 do Conselho Nacional de Saúde (Brasil, 2012a).

Para essa etapa do estudo, foi realizado um estudo exploratório, descritivo de abordagem qualitativa (Gil, A. C., 2017) em Campina Grande - PB, município com uma população de 407.472 habitantes, localizado na mesorregião do agreste paraibano e segundo mais desenvolvido do Estado, concentrando 14,1\% do total das riquezas produzidas na Paraíba (Brasil, 2020).

A população do estudo foi constituída por profissionais de ensino superior da ESF envolvidos com a assistência dos casos de TB e lotados em Unidades Básicas de Saúde (UBS) da zona urbana do município.

Para seleção da amostra - por conveniência -, optou-se por integrantes de Equipes que atendessem em territórios que costumam ter pessoas em situação de rua, a partir de um levantamento previamente realizado com a Equipe de Consultório na Rua (ECR) do município. Das 27 equipes selecionadas, 45 profissionais aceitaram participar dessa etapa da pesquisa.

Participaram do estudo médicos, enfermeiros e odontólogos, além dos profissionais do Núcleo Ampliado de Saúde da Família e Atenção Básica (NASF-AB), que atuam na assistência à TB. Como critério de inclusão elegeu-se aqueles profissionais em efetivo exercício e que estavam presentes na UBS no momento da visita do pesquisador para a realização da coleta de dados.

Para a coleta de dados, ocorrida entre abril a julho de 2017, foi realizada uma entrevista mediada por um roteiro semiestruturado para apreender as representações sociais dos profissionais sobre aspectos envolvidos na assistência ao portador de TB em situação de rua. Para tanto, considerou-se a organização da rede atenção à saúde, o conhecimento acerca da PSR, as ações desenvolvidas para o controle da TB e os fatores que pudessem representar obstáculos para o atendimento. Os 
participantes do estudo responderam também a um questionário para caracterização dos sujeitos, das Unidades de Saúde e da assistência à TB, com ênfase na PSR. O profissional que concordou em participar da pesquisa assinou o Termo de Consentimento Livre e Esclarecido. A aplicação dos instrumentos ocorreu nas UBS, em locais que resguardaram a privacidade dos entrevistados, com duração média de 38 minutos.

Sobre a análise dos dados, inicialmente foi realizada a análise descritiva para apresentação das características demográficas e sociais dos sujeitos da pesquisa, dos serviços de saúde e da assistência à TB - ênfase na PSR - com a obtenção de frequências absolutas e relativas e cálculos das médias, de acordo com o tipo de cada variável pesquisada. Essa análise foi realizada com o auxílio do pacote estatístico Stata, versão 12.

As entrevistas compuseram o corpus textual que foi transcrito para um arquivo no formato .txt sem formatação. Cada texto (entrevista) foi devidamente lido e alinhado para eliminar inconsistência - como vícios de linguagem - por dois pesquisadores. Para preservar o anonimato dos participantes, foi utilizada a categoria profissional, seguida de um número aleatório entre 1 e 45.

Os dados das entrevistas foram organizados e analisados com o auxílio do software de análise textual Iramuteq (Interface do software R para análise multidimensional de textos e questionários). Foi realizada a análise lexical de dados textuais, com análise lexicográfica básica (cálculo da frequência de palavras) e multivariada (Classificação Hierárquica Descendente, método de Reinert).

Para garantir aproveitamento dos dados da forma mais fidedigna possível ao coletado nas entrevistas, adotou-se a parametragem padrão do Iramuteq para as palavras ativas e suplementares consideradas na análise dos dados, onde nenhuma forma é eliminada.

As classes obtidas com a realização da Classificação Hierárquica Descendente (CHD) foram nomeadas e analisadas considerando os segmentos de textos e as palavras estatisticamente significativas mais relevantes para a caracterização de cada classe, de acordo com o teste qui-quadrado. Importante ressaltar que este teste considera significativos ( $\mathrm{p}$ valor $<0,05$ ) resultados a partir de 3,84; quanto maior o valor, portanto, mais significativa é a palavra para a composição de cada classe.

A análise utilizou o dispositivo teórico-analítico das representações sociais (RS), que compreende um conjunto de conceitos, proposições e explicações que são criadas na vida cotidiana, mediada pela comunicação de grupos sociais. Seu estudo possibilita realizar uma análise de processos em que os indivíduos, ao interagirem socialmente, constroem teorias sobre objetos sociais que viabilizam a comunicação e a organização de comportamentos e práticas que se alimentam tanto de teorias científicas quanto de dimensões culturais e ideológicas, de experiências e comunicação cotidiana. Dessa forma, viabilizam a leitura de fenômenos, permitem construir modelos explicativos para os problemas estudados e evidenciam diferentes estratégias de enfrentamento (Moreira, \& Jesuino, 2003).

\section{Resultados e Discussão}

Participaram do estudo profissionais de cinco distritos sanitários da zona urbana de Campina Grande; os distritos um, três e cinco tiveram os maiores percentuais de participantes com 22,2\%, 28,9\% e 28,9\%, respectivamente. A maioria dos profissionais trabalha 08 horas por dia $(86,7 \%$, média de 7,8 horas), é do sexo feminino $(95,6 \%)$, enfermeiros $(53,3 \%)$ na faixa etária de 31 a 40 anos (31,1\%), média de idade de 45,7 anos. Entre os entrevistados, a maioria foi constituída de profissionais concursados (71,1\%), com mais de 5 anos de atuação no serviço (75,5\%, média de 9,2 anos), mais de 10 anos de formação (77,8\%, média de 20 anos) e com pós-graduação relacionada ao trabalho desenvolvido na ESF (66,7\%).

Sobre as características da assistência à TB com ênfase na PSR, essa população estava presente em 35,5\% dos territórios de atuação dos profissionais no momento da entrevista, sendo que 48,9\% informaram que as equipes que integram já 
atenderam esse público. Deve-se destacar que 37,8\% dos profissionais não souberam informar se havia, no momento da entrevista, pessoas em situação de rua no território de atuação da equipe. Para o atendimento dos casos de TB, 44,4\% relataram a participação de todos os integrantes da equipe básica e 40,0\% relataram, inclusive, a participação de profissionais da Equipe de Saúde Bucal e do NASF.

Além disso, 44,5\% dos profissionais afirmaram ter pacientes com TB no território no momento da entrevista. O atendimento à pessoa com TB e a identificação de SR foram relatados por 91,1\% e 95,6\% dos profissionais, respectivamente. Ressalta-se que mais de 40,0\% dos entrevistados não souberam informar se havia casos de SR ou de TB em pessoas em situação de rua no território no momento da entrevista. Um percentual de $15,6 \%$ dos entrevistados relatou ter ocorrido identificação de SR e 22,2\%, a ocorrência de atendimento à TB na PSR pelas respectivas equipes que integram.

O corpus textual, composto por 45 textos, foi submetido à CHD, que fez a segmentação do corpus e classificação dos segmentos de texto em função dos vocábulos evocados, análise da lexicografia das palavras e o alinhamento destas em classes. Dessa forma, dos 1.512 segmentos textuais iniciais, houve um aproveitamento de 1.410 segmentos $(93,25 \%)$, emergindo 49.656 ocorrências (palavras, vocábulos), sendo 3.889 formas distintas e 1.850 com apenas uma ocorrência. Importante ressaltar que na CHD, para que sua análise seja útil, deve ocorrer uma retenção mínima ou aproveitamento de, pelo menos, 75,0\% dos segmentos de texto (Camargo, \& Justo, 2018).

O conteúdo analisado do corpus textual foi categorizado em quatro classes: Classe 1, com 200 segmentos textuais (14,18\%, dos segmentos aproveitados); classe 2, com 488 segmentos (34,61\%); Classe 3, com 492 segmentos (34,89\%); e a classe 4, $230(16,31 \%)$.

Como pode ser observado no dendrograma (Figura 1), o corpus foi dividido em dois subcorpus. O primeiro, constituído pela classe 1 , está relacionado a todo um contexto de vulnerabilidade ocasionado pela vida nas ruas e a consequente suscetibilidade a uma série de doenças e agravos à saúde que a PSR está exposta. O primeiro originou a classe 2 e, posteriormente, as classes 3 e 4; esse subcorpus trata da dificuldade de acesso da PSR à APS, com os mais variados obstáculos existentes e a dificuldade de controle da TB nessa população, com as ações direcionadas para o diagnóstico e tratamento da doença. No dendrograma, é possível observar também as palavras mais significativas e relevantes evocadas pelos profissionais para a constituição de cada classe, selecionadas a partir do teste qui-quadrado. 
Figura 1: Dendrograma - Principais palavras evocadas por classe: representações sociais de profissionais da APS sobre o controle da TB na PSR, Campina Grande, PB.

\begin{tabular}{|c|c|c|c|c|c|c|c|c|c|c|c|}
\hline & \multicolumn{8}{|c|}{ Corpus: 45 textos } & & & \\
\hline & \multicolumn{8}{|c|}{1512 Seguimentos Textuais } & & & \\
\hline & \multicolumn{8}{|c|}{ Segmentos Textuais aproveitados: $1410(93,25 \%)$} & & & \\
\hline \multicolumn{3}{|c|}{$\begin{array}{l}\text { Classe 1: A vida nas Ruas: } \\
\text { Contexto de vulnerabilidade }\end{array}$} & \multicolumn{3}{|c|}{$\begin{array}{c}\text { Classe 2: Desafios para o } \\
\text { diagnósticoe tratamento da TB } \\
\text { na PSR }\end{array}$} & \multicolumn{3}{|c|}{$\begin{array}{c}\text { Classe } 3 \text { : Obstáculos para o } \\
\text { acesso da PSR à APS }\end{array}$} & \multicolumn{3}{|c|}{ Classe 4: A problemática da ECR } \\
\hline Palavras & $\mathrm{n}$ & $x^{2}$ & Palavras & $\mathrm{n}$ & $x^{2}$ & Palavras & $\mathrm{n}$ & $x^{2}$ & Palavras & $\mathbf{n}$ & $x^{2}$ \\
\hline Moradia & 28 & 157,75 & Medicação & 71 & 133,52 & Barreiras & 42 & 55,02 & ECR & 47 & 149,35 \\
\hline Social & 30 & 150,86 & Tratamento & 100 & 111,10 & Achar & 119 & 45,27 & Conhecimento & 30 & 83,31 \\
\hline Droga & 28 & 97,96 & Tomar & 46 & 75,44 & EqSF & 60 & 41,26 & Ouvir & 16 & 65,04 \\
\hline Violência & 14 & 71,46 & Referência & 49 & 63,85 & Atender & 71 & 37,35 & Nunca & 34 & 64,68 \\
\hline Vulnerabilidade & 14 & 48,24 & Exame & 52 & 62,03 & Seviço & 47 & 28,16 & Trabalho & 35 & 54,93 \\
\hline Higiene & 10 & 47,54 & Vir & 89 & 63,22 & Profissional & 40 & 27,85 & Especifico & 15 & 40,96 \\
\hline Familia & 30 & 36,87 & Pedir & 37 & 46,18 & Existir & 58 & 20,67 & Projeto & 10 & 39,82 \\
\hline Fome & 5 & 30,36 & Marcar & 15 & 28,64 & Procurar & 42 & 20,44 & Participar & 9 & 34,85 \\
\hline Exposição & 5 & 30,36 & Terminar & 13 & 24,79 & RAS & 22 & 19,94 & Desenvolver & 12 & 30,96 \\
\hline Apoio & 13 & 26,09 & Diagnóstico & 13 & 24,79 & Território & 22 & 16,52 & Contato & 17 & 23,11 \\
\hline Frio & 4 & 24,27 & Raio-X & 12 & 22,87 & Organizar & 13 & 15,31 & Palestra & 4 & 20,58 \\
\hline Adoecimento & 4 & 24,27 & Baciloscopia & 12 & 22,87 & Adscrever & 10 & 15,31 & Funcionamento & 4 & 20,58 \\
\hline Marginalidade & 5 & 23,67 & Solicitar & 12 & 19,30 & Invisivel & 6 & 8,0 & Confessar & 4 & 20,58 \\
\hline Emocional & 3 & 18,19 & Encaminhar & 26 & 18,21 & Demanda & 21 & 7,99 & Abordagem & 6 & 14,08 \\
\hline Álcool & 6 & 17,37 & Continuidade & 9 & 17,11 & Itinerante & 50 & 7,48 & Reunião & 6 & 14,08 \\
\hline \multicolumn{3}{|c|}{200 ST $(14,2 \%)$} & \multicolumn{3}{|c|}{488 ST $(34,6 \%)$} & \multicolumn{3}{|c|}{492 ST $(34,9 \%)$} & \multicolumn{3}{|c|}{230 ST $(16,3 \%)$} \\
\hline
\end{tabular}

Fonte: Dados da pesquisa.

Na classe 1 - A vida nas ruas: contexto de vulnerabilidade - destacaram-se as palavras: moradia, social, vulnerabilidade, exposição, droga, familiar, violência, higiene, fome, apoio, frio, marginalidade, adoecimento, emocional e álcool (Figura 1). Tais elementos evidenciam as características e adversidades a que a PSR está exposta. Toda essa problemática com que a PSR convive também pode ser constatada nos trechos a seguir:

Não tem um lar como a gente diz, não tem um domicílio fixo e a gente sempre encontra ela perambulando, marginalizada. Todo tipo de exposição de todos os riscos a todas as doenças. Infecções sexualmente transmissíveis que a gente sabe que é uma população com altíssimo risco de ter e as doenças em geral, as parasitoses, as verminoses, as arboviroses aumento do risco por eles estarem expostos, as infectocontagiosas já que é uma população que, em geral, não tem nenhum atendimento de saúde e um desenvolve as doenças e vai contagiando os demais e fica aquele grupo todo sem atendimento e sem tratamento. (Participante 32 - Médica, até 05 anos de atuação na APS, contratada)

Eles estão vulneráveis porque estão suscetíveis a risco social, a risco de doença, eles não têm cuidados, muitas vezes têm seu direito negado à saúde, à educação, à orientação, à informação, ou seja, o que eu percebo na PSR é que eles estão negados a todos os seus direitos, estão em risco social, familiar e estão vulneráveis também, marginalizados. (Participante 31 - Assistente Social, entre 06 e 10 anos de atuação na APS, concursada) 
A análise dos relatos evidencia que não ter uma moradia fixa e possuir vínculos familiares rompidos ou fragilizados são os principais pontos que caracterizam e interferem na qualidade de vida da PSR. Ademais, repousam sobre a referida população preconceitos e estigmas, os quais, no entender de Hino, Monroe, Takahashi, Souza, Figueiredo, e Bertolozzi (2018), conferem àquelas pessoas um lugar de desprestígio social e a vulnerabilizam a condições deletérias de vida. Importa enfatizar que a questão social de uma perspectiva macro é marcante, de tal modo que os profissionais percebem que, além do não atendimento às necessidades de saúde, também há a privação de vários aspectos, como segurança física, alimentar, emprego/renda, falta de higiene, exposição ao calor, frio, uso de drogas, ou seja, vislumbram um problema estrutural na assistência como um todo, o que torna a PSR susceptível a uma série de doenças e agravos à saúde (Silva, Viana, Almeida, Santos, \& Nery, 2021; Hino, Monroe, Takahashi, Souza, Figueiredo, \& Bertolozzi, 2018).

Essa fração da população encontra-se econômica, sanitária e nutricionalmente desprotegida e, portanto, mais suscetível a doenças como a TB, condição que limita ainda mais as possibilidades de trabalho e de manutenção do sustento, bem como impacta na possibilidade de se encontrar um local adequado para o repouso, agravada pela, quase sempre, inexistência de uma rede de apoio familiar eficiente para o incentivo de manutenção do tratamento, gerando um círculo vicioso que torna a PSR ainda mais vulnerável.

A classe 2, desafios para o diagnóstico e tratamento da TB na PSR, teve como principais palavras: medicação, tratamento, tomar, referência, exame, vir, pedir, marcar, terminar, diagnóstico, raio-x, baciloscopia, solicitar, encaminhar, continuidade (Figura 1).

Nessa classe, são elencadas as ações para o controle da TB na PSR, com destaque para o diagnóstico e tratamento da doença. Sobre o diagnóstico, os relatos mostram que, no geral, as especificidades da PSR não são consideradas e os profissionais procedem da mesma forma que com a população geral, dificultando, assim, o controle da TB na PSR. É destacada também a importância do trabalho dos ACS na busca ativa e identificação dos sintomáticos respiratórios.

O nosso trabalho é organizado assim, a gente tem, segue aquele protocolo da pessoa que chega aqui com sintomático respiratório para a gente fazer o exame, orientar aonde ele vai fazer, para onde ele vai, depois vai para referência e volta para a gente dar, continuar ao tratamento. (Participante 19 - Médica, entre 6 e 10 anos de atuação na APS, concursada)

Através dos ACS em uma busca ativa, acho que seria o passo inicial, se nós tivermos essa PSR, íamos pedir para os ACS fazer a busca ativa e trazer esses pacientes, solicitar os exames e os encaminhar para a referência. (Participante 44 - Enfermeira, entre 06 e 10 anos de atuação na APS, concursada)

Segundo recomendações do MS, o preconizado à PSR é que todas as pessoas que apresentem tosse, independentemente do tempo, ou radiografia torácica sugestiva para TB realizem a coleta de escarro para baciloscopia ou teste rápido molecular e teste de sensibilidade de forma imediata já na UBS. Para a PSR, é essencial o acolhimento com uma abordagem rápida para o diagnóstico da TB e garantia de tratamento até o fim. Deve-se destacar que a tosse, mesmo que inferior a três semanas, já constitui indicativo de atenção para TB pulmonar. Vale salientar, ainda, que a PSR, muitas vezes, não tem a percepção exata do tempo e, pelo fato de viver em um clima favorável ao surgimento de doenças respiratórias ou em virtude da dependência química, tende a desvalorizar o sintoma existente, culminando em um diagnóstico tardio, podendo levá-la a desenvolver a forma mais resistente da TB (Brasil, 2019b).

Por se tratar de uma doença transmissível, o diagnóstico da TB deve ser realizado da forma mais rápida possível para que se possa intervir na cadeia de transmissão da doença, sendo imprescindível a responsabilização da APS nessa etapa do processo. Porém, o que se observa é que os profissionais desse nível de atenção apresentam dificuldades para identificar os SR e realizar coleta de material para a baciloscopia. Essa atividade deveria ser realizada em todas as situações e tendo a rede de 
atenção à saúde preparada para realização da baciloscopia, tendo em vista que possibilita o diagnóstico da TB mesmo que o SR não retorne ao serviço de saúde (Santos, Brufentinker, Pena, Saraiva, \& Boing, 2021).

Os relatos indicam que, contrariando as recomendações do Ministério da Saúde, o diagnóstico da TB é centralizado, inclusive para a coleta de material, o que, praticamente, inviabiliza a realização pela PSR, pois a situação social em que se encontra faz com que priorize outros elementos em detrimento aos relacionados à prevenção, diagnóstico e tratamento da doença (Zuim, \& Trajman, 2018).

Acerca da realização do tratamento da TB na PSR, os profissionais entrevistados fizeram menção a uma diversidade de representações, que vão desde a inviabilidade, perpassando pelos desafios - dadas as características da PSR e a elevada taxa de abandono ao tratamento -, até a adoção de estratégias direcionadas, considerando as peculiaridades desse grupo vulnerável.

Possivelmente, isso não vai funcionar direito porque, geralmente, essas pessoas em situação de rua são pacientes de saúde mental, geralmente, eles não compreendem ou rejeitam, primeiro, fazer o exame, segundo o tratamento. Eu acho que, para tuberculose e para hanseníase, pelo menos Campina Grande, temos acesso ao tratamento bem direito, todo mês, mas, nesse caso de pessoa em situação de rua, primeiro, ele analisar e aceitar o tratamento, e tomar nos horários certos, tomar a dose certa vai ser bem difícil. (Participante 42 - Médica, até 05 anos de atuação na APS, contratada)

Olha como é difícil, todos os dias o porteiro ia lá atrás dele, trazia lá na UBS e a gente dava o remédio, aí dava o copo com leite e o pão para ele tomar a medicação e estar, de certa forma, com o estômago forrado. Então assim, pelo fato da gente ficar aqui dentro, não estar na rua, já que eles ficam nessa, como itinerante por aí, agora está aqui, depois está em outro lugar, então fica difícil da gente meio que fazer essa busca ativa. Eu não tenho conhecimento de nenhuma proposta até hoje das EqSF que eu trabalho, voltada para esse público. (Participante 15 Assistente Social, entre 06 e 10 anos de atuação na APS, contratada)

É válido lembrar que o tratamento da TB dura no mínimo seis meses, sendo gratuito, exclusivamente oferecido pelo SUS e, preferencialmente, realizado na APS pela estratégia do Tratamento Diretamente Observado (TDO). Nesse contexto, não apenas os profissionais da saúde podem operacionalizar o TDO. Outros profissionais - assistentes sociais, vigilantes, recepcionistas, entre outros -, desde que capacitados e supervisionados por profissionais de saúde, também podem operacionalizar o TDO. Além disso, é imprescindível a identificação e realização de parcerias com instituições governamentais e não governamentais (abrigos, albergues, centros de convivência, etc.) que possam contribuir com a realização do tratamento da TB na PSR (Brasil, 2019b).

O manual de recomendações para o controle da TB na PSR assinala a importância decisiva da parceria intersetorial com vistas à prevenção, detecção, diagnóstico e tratamento dessa enfermidade (Brasil, 2012b). Nessa perspectiva, Barbosa, A. D. (2019) sustenta que a articulação das instituições que configuram a rede de atendimento com as UBS é indispensável para garantir acesso, adesão e continuidade do tratamento da TB na PSR. Tais equipamentos promovem serviços que englobam capacitação profissional, atenção psicossocial, acolhimento, abrigo, tratamento hospitalar, ações articuladas com a ECR, assistência social, entre outros.

A literatura consultada destaca ainda estudos desenvolvidos em países como Alemanha, EUA, Brasil, Haiti, os quais atestaram que a PSR necessita de um serviço de saúde pública articulado e eficiente para lidar com suas demandas, que são diferentes daquelas caracterizantes da população em geral (Onwubiko, Wall, Sales, \& Holland., 2019; Hino, Monroe, Takahashi, Souza, Figueiredo, \& Bertolozzi, 2018; Riquelme, Morales, Aguilera, Espinoza, Vidal, \& Riquelme, 2018; Queiroga, SÁ, \& Grazzinelli, 2018).

Na classe 3, Obstáculos para o acesso da PSR à APS, chamam atenção as palavras: barreira, achar, EqSF (Equipe de Saúde da Família), atender, serviço, profissional, existir, procurar, RAS, território, organizar, adscrever, invisível, demanda e 
itinerante (Figura 1); atreladas a uma série de empecilhos informados pelos profissionais que dificultam, quando não chegam a impedir, que a PSR possa ser atendida na APS.

Entre os obstáculos, foram evidenciados elementos como o desconhecimento da PSR no território, de como atender essa população e da organização da RAS, como pode ser observado nos trechos a seguir:

A primeira barreira que eu acho é o desconhecimento da EqSF com relação à pessoa em situação de rua, eles não conhecem, não sabem que atitude, a grande maioria dos profissionais da EqSF não tem assim o conhecimento, que atitude, como fazer para minimizar aquela situação ou dar um encaminhamento, acho que essa é a primeira barreira. (Participante 10 - Assistente social, mais de 10 anos de atuação na APS, concursada)

Eu estou com um caso na área de uma pessoa nessa situação e assim, já conversei com o CRAS, assistente social, mas nada resolve. Acho que uma das barreiras é a gente não ter resolutividade. A gente não tem uma organização e o principal que é que aquilo seja resolutivo, que a gente consiga realmente resolver aquele problema. Me sinto, às vezes, de mãos atadas [...] (Participante 23 - Enfermeira, entre 6 e 10 anos de atuação na APS, contratada)

No cuidado à PSR é importante que os profissionais da saúde levem em consideração as características relacionadas aos hábitos de vida específicos desse grupo, assim como as barreiras para acesso aos serviços de saúde e de assistência social que dificultam o controle de doenças como a TB (Santos, Brufentinker, Pena, Saraiva, \& Boing, 2021; Hino, Monroe, Takahashi, Souza, Figueiredo, \& Bertolozzi, 2018). Considerando as características dessa população, que possui percepção do adoecimento e exige cuidados com a saúde peculiares, além de estarem em risco ou acometidos pela TB, o MS recomenda que os serviços adotem uma série de ações específicas a essa população para ampliar a efetividade no controle da doença, principalmente pelos serviços da APS (Brasil, 2012b; Brasil, 2019b).

Dessa forma, o desafio de controlar a TB perpassa a superação de fragilidades como as relacionadas à capacitação dos profissionais. A falta de recursos humanos qualificados é apontada como um dos fatores responsáveis pela limitada incorporação das ações preconizadas pelo MS para o controle da TB pelos serviços da APS (Wysocki, Ponce, Brunello, Beraldo, Vendramini, Scatena, Netto, \& Villa, 2017).

Contribuindo com as fragilidades apontadas, em uma completa deturpação conceitual, a territorialização, a adscrição da clientela e a falta de documentos também são elencadas como elementos que dificultam ou impedem o acesso da PSR à APS:

[...] a gente tem uma área de abrangência fechada e o profissional não querer atender esse usuário se ele não tiver dentro da área de abrangência, isso é uma barreira, mas é uma barreira necessária, porque se abrir a gente vai ser Centro de Saúde, não vai ser APS, a gente vai sair atendendo todo mundo [...] (Participante 12 - Enfermeira, entre 6 e 10 anos de atuação na APS, concursada)

[...] primeiro porque o usuário na APS hoje em dia tem o cartão do SUS e eles não têm. Infelizmente, é uma barreira. Muitas vezes aqui eu brigo até com a médica, chega umas intercorrências aqui e ela já pergunta cadê o cartão do SUS, eu digo que não tem e ela fala que não vai atender sem cartão do SUS [...] (Participante 32 - Enfermeira, entre 6 e 10 anos de atuação na APS, concursada)

[...] a barreira aqui é enorme. Como é que uma pessoa que não tem documento, que não tem nada, não tem cartão do SUS, não tem onde ficar, hoje ele vem, amanhã ele não vem, a principal barreira é isso, não tem vínculo, a população não tem vínculo, esses pacientes não têm vínculo com nada [...] (Participante 43 - Médica, até 5 anos de atuação na APS, contratada)

A Política Nacional de Atenção Básica (PNAB) determina que população adscrita é aquela "presente no território da UBS, de forma a estimular o desenvolvimento de relações de vínculo e responsabilização entre as equipes e a população, 
garantindo a continuidade das ações de saúde e a longitudinalidade do cuidado e com o objetivo de ser referência para o seu cuidado" (Brasil, 2017b, p.1).

A exigência de moradia fixa no território da UBS não deveria, portanto, configurar como elemento para impedir a inserção da PSR na RAS, pois tal população não dispõe dessa forma de fixação no território. Vale destacar que a PNAB traz a territorialização e a adscrição da clientela como elementos de destaque para a organização do serviço da APS e determina:

A Política Nacional de Atenção Básica tem na Saúde da Família sua estratégia prioritária para expansão e consolidação da Atenção Básica... considera e inclui as especificidades locorregionais, ressaltando a dinamicidade do território e a existência de populações específicas, itinerantes e dispersas, que também são de responsabilidade da equipe enquanto estiverem no território, em consonância com a política de promoção da equidade em saúde (Brasil, 2017 b, p. 1).

Sobre a exigência do Cartão Nacional de Saúde (CNS) no atendimento realizado pela APS, Barbosa (2018), em estudo realizado na capital paraibana, concluiu que a inexistência do CNS se delimita enquanto uma barreira substancial para o atendimento da pessoa em situação de rua nas unidades de saúde pública, indicando uma carência do próprio sistema em sanar essa dificuldade, na medida em que vai impossibilitar o atendimento, diagnóstico e até mesmo tratamento de doenças como a TB.

Visto isso, deve-se destacar que a Portaria Ministério da Saúde/GM n 940 de 2011, que regulamenta o Sistema do CNS, no seu artigo 13, determina que não é impedimento para o atendimento não ter CNS, desconhecer o número do CNS ou impossibilidade de cadastramento à Base Nacional de Dados dos Usuários das Ações e Serviços de Saúde (Brasil, 2011a).

Fica claro que é papel do próprio sistema de saúde promover acessibilidade aos mecanismos criados para controlar sua organização, isto é, se a RAS institui normas para nortear o funcionamento dos serviços, é também seu papel garantir que seus profissionais conheçam essas normas como forma de universalizar a acessibilidade aos serviços para seus usuários.

Por fim, destacou-se também, como elementos que representam barreiras para o acesso à APS, as precárias condições de higiene e estigmas como a violência que, por vezes, são atrelados à PSR.

[...] às vezes, é um paciente que cheira mal, que ele vem mal vestido, que ele vem com mal cheiro mesmo que a gente se afasta, que o outro diz: "fulano está fedendo", se sentar na cadeira, vai sujar a cadeira do profissional, e aí é assim, junta isso tudo e isso aí é barreira. E ele mesmo se sente assim, eu não sou dali não, eu não faço parte daquela UBS não, eu sou assim, não sou digno de ali ser atendido [...] (Participante 04 - Enfermeira, entre 6 e 10 anos de atuação na APS, concursada)

A violência atrelada à PSR gera uma barreira para que se tenha acesso, para que o ACS traga essa informação para a UBS [...] (Participante 05 - Dentista, entre 6 e 10 anos de atuação na APS, concursada)

A PSR é um estrato social que normalmente está em situação limite de pobreza, que se encontra revestido por um manto de conceitos pré-estabelecidos que lhe confere um lugar social marcado pelo preconceito e estigmatização. Ao mesmo tempo, estão vulneráveis a uma condição de vida insalubre, na qual se deparam com diversos problemas, como falta de emprego, falta de alimentação saudável, falta de higiene, exposição às intemperes climáticas, compartilhamento de seringas, baixa imunidade, nomadismo característico por sua situação econômica e social, uso de substâncias psicoativas e ausência de moradia fixa, além da manutenção penosa desse ciclo de vida (Barbosa, 2018; Hino, Monroe, Takahashi, Souza, Figueiredo, \& Bertolozzi, 2018).

No estudo de Barbosa (2018), evidenciou-se que o preconceito afeta, inclusive, a busca por atendimento em saúde por parte da própria PSR, que acredita não merecer e que não vai ser atendida na unidade. Dessa forma, a busca por atendimento às demandas em saúde é relegada ou até mesmo suprimida das necessidades que consideram indispensáveis. Ressalta-se que os 
profissionais de saúde têm o dever de agir seguindo os princípios do SUS, as políticas e diretrizes destinadas à PSR, isto é, garantindo acessibilidade ao serviço de saúde sem qualquer forma de acepção entre as pessoas. A Política Nacional para a PSR delimita objetivos que visam justamente diminuir essas desigualdades, garantindo acesso amplo, simplificado e seguro aos serviços de saúde (Brasil, 2009).

Importante destacar que as barreiras relatadas não estão presentes em todas as equipes. Foram observados também profissionais que reconhecem a PSR como usuária do serviço e que este está de portas abertas para o atendimento, com destaque para a atuação dos ACS em identificar a PSR no território e aproximar os demais integrantes da equipe dessa população.

Eu não posso dizer que tenha barreiras, eu acredito que exista, mas eu vou pela minha situação, eu não ofereço a barreira, entendeu? Se ele vier aqui, ele vai ser atendido, então eu acredito que deva ser assim em todas as UBS. (Participante 08 - Enfermeira, entre 6 e 10 anos de atuação na APS, concursada)

Imagino que quando tiver alguma pessoa em situação de rua o primeiro que tem que saber é o ACS, o ACS passa para a EqSF, a EqSF é obrigada ir até onde está a pessoa em situação de rua, se ele não quer vir até a UBS, a gente vai até ele para fazer o que for necessário para garantir a saúde dessa pessoa. (Participante 42 - Médica, até 5 anos de atuação na APS, contratada)

Os elementos observados na classe 3 atestam como o serviço ainda é incipiente à problemática da PSR. As deficiências perpassam por representações sociais inerentes à organização do serviço, capacitação profissional, até as questões pessoais, em atitudes carregadas de uma conotação preconceituosa que agravam o contexto da PSR. Não bastasse toda a adversidade presente pelo próprio contexto da rua, o serviço de saúde, muitas vezes, contribui para tornar mais grave a situação dessa população vulnerável, que deveria ser priorizada no atendimento.

De forma atrelada e complementando aspectos observados na classe 3, a classe 4 - A problemática da ECR - parece intensificar as barreiras para o acesso da PSR à APS. Essa classe é constituída pelas palavras: ECR, conhecimento, ouvir, nunca, trabalho, específico, projeto, participar, desenvolver, funcionamento, contado, confessar, abordagem, palestra, reunião (Figuras 1).

Contrariando o propósito da ECR, os profissionais, em sua maioria com pessoas em situação de rua no território, relataram desconhecer a existência dessa equipe no município de Campina Grande. Ao serem indagados sobre a ECR, muitos afirmaram que conheciam, mas que isso estava apenas no campo teórico ou só de um "eu ouvi falar". Relataram também não saber do funcionamento e dificuldades em desenvolver atividades em parceria, como pode ser observado nos trechos a seguir:

[...] em Campina Grande, que eu saiba, não, se existe a ECR, eu não conheço. (Participante 41 - Assistente Social, entre 6 até 10 anos de atuação na APS, concursada)

Conheço, já ouvi falar, mas do trabalho não tenho propriedade do assunto. Já ouvi falar na mídia, mas não sei como funciona exatamente o serviço. Já ouvi falar que existe aqui em Campina Grande, mas através da mídia. (Participante 44 - Enfermeira, entre 6 e 10 anos de atuação na APS, concursada)

[...] a gente não teve nenhum contato com esse pessoal para saber como é desenvolvido o trabalho deles, da ECR, a gente sabe que tem esse serviço, mas a gente não sabe o que é que eles querem, o que é que precisa fazer, a gente não tem, não foi comunicado a ninguém que existe, é um serviço que está funcionando, mas a gente da APS a gente não sabe [...] (Participante 12 - Enfermeira, entre 6 até 10 anos de atuação na APS, concursada)

Alguns profissionais relataram, inclusive, não ter o conhecimento da ECR enquanto estratégia de atendimento da PSR instituída pela PNAB. A ECR foi uma das principais formas de universalizar o atendimento à PSR, tendo em vista as demandas específicas dessa população. Essa modalidade de atenção foi criada por meio da portaria n ${ }^{\circ} 122$ de 25 de janeiro de 
2011 com o objetivo de garantir atenção integral em saúde à PSR, sendo um serviço da APS e composto por uma equipe multiprofissional (Brasil, 2011b).

As ECR têm por objetivo ampliar o acesso da PSR ao serviço de saúde, ofertando atenção de forma integral a esse grupo, com a criação de laços de longitudinalidade entre equipe e paciente. O trabalho da ECR é realizado de forma itinerante, visando à necessidade de se adequar às demandas da PSR, podendo ocorrer em período diurno ou noturno, em todos os dias da semana, priorizando o cuidado mais próximo ao paciente e em articulação com outros serviços, principalmente com as demais equipes da APS (Brasil, 2017b).

Contudo, os relatos observados nessa classe atestam a insipiente atuação da ECR no cenário investigado. A ECR deveria ser umas das protagonistas no cuidado à PSR de Campina Grande, ampliando o acesso dessa população à APS, trabalhando em parceria com as demais equipes, capacitando os profissionais e realizando o matriciamento para potencializar e aumentar a resolutividade das ações desenvolvidas.

\section{Conclusão}

O estudo realizado evidenciou um conjunto de representações sociais de profissionais que atuam na APS do município de Campina Grande sobre a PSR e sobre o acometimento dessa população pela TB que influenciam diretamente nas ações realizadas para o controle da doença. Observou-se que essas representações estão relacionadas ao processo de formação/capacitação dos profissionais e à forma como a Rede de Atenção à Saúde do Município está organizada para prestar assistência a esse grupo populacional vulnerável. Vulnerabilidade essa tão bem representada nos relatos dos profissionais, ao reconhecerem as características dessa população que a tornam susceptível a uma série de doenças, condições e agravos à saúde. Trata-se de um conjunto de características desfavoráveis que resultam em uma condição de vida precária, insalubre.

O reconhecimento da vulnerabilidade da PSR e maior susceptibilidade ao adoecimento não resultam, no entanto, em uma priorização no atendimento ou cuidado diferenciado na da APS. Foi evidenciada no estudo uma série de barreiras impostas à PSR que tornam a sua condição ainda mais complexa.

Sobre a organização dos serviços e ações desenvolvidas, prevaleceram representações que consideram a assistência à TB norteada por um único formato/protocolo que não considera as especificidades, contextos e vulnerabilidades como as vivenciados pela PSR, inclusive sem considerar as recomendações do próprio MS. Observa-se que, no geral, as especificidades da PSR não são consideradas e os profissionais procedem da mesma forma que com a população geral, dificultando, assim, o controle da TB na PSR.

Nesse contexto, a atuação da ECR, que deveria romper com as barreiras para o acesso e qualificar as ações voltadas ao cuidado à PSR, mostrou-se seriamente comprometida pelos relatos dos profissionais de falta de capacitação, parceria, chegando ao desconhecimento da existência dessa modalidade de atendimento no município de Campina Grande.

Portanto, espera-se que as fragilidades elencadas, evidenciadas nas representações sociais de profissionais da APS que se configuram como problemas para o controle da TB na PSR, possam servir de subsídios para repensar a assistência à PSR de Campina Grande, com o desenvolvimento de estratégias de intervenção que subsidiem o adequado cuidado dessa população no contexto SUS.

Por fim, que os achados dessa pesquisa possam servir de subsídio e instigar a realização de novos estudos sobre como a organização dos serviços, a atuação dos profissionais de saúde e o contexto de vida de populações vulneráveis influenciam tanto na ocorrência com na capacidade de resposta para o controle de doenças tão sensíveis a esses contextos como a TB. 


\section{Agradecimentos}

O presente trabalho foi realizado com apoio do CNPq, Conselho Nacional de Desenvolvimento Científico e Tecnológico - Brasil.

\section{Referências}

Barbosa, A. D. (2019). Tuberculose na População em Situação de Rua: desafios para o cuidado em saúde. Especialização em Saúde Pública, Escola de Saúde Pública do Estado de Minas Gerais, Belo Horizonte, MG, Brasil.

Barbosa, K. K. S. (2018). Significados e experiências de pessoas em situação de rua acometidas por tuberculose. 121f. Tese de Doutorado em Enfermagem, Centro de Ciências da Saúde, Universidade Federal da Paraíba, João Pessoa, PB, Brasil.

Brasil. Decreto $n^{\circ} 7.053$, de 23 de setembro de 2009. (2009). Institui a Política Nacional para a População em Situação de Rua e seu Comitê Intersetorial de Acompanhamento e Monitoramento e dá outras providências. Recuperado em 02 de dezembro, 2020, de http://www.planalto.gov.br/ccivil_03/_ato20072010/2009/decreto/d7053.htm.

Brasil. Ministério da Saúde. (2011a). Portaria $N^{\circ}$ 940, de 28 de abril de 2011: Regulamenta o Sistema Carteira Nacional de Saúde. Recuperado em 10 de outubro, 2020, de https://bvsms.saude.gov.br/bvs/saudelegis/gm/2011/prt0940_28_04_2011.html.

Brasil. Ministério da saúde. (2011b). Portaria $N^{o}$ 122, de 25 de janeiro de 2011: Define as diretrizes de organização e funcionamento das Equipes de Consultório na Rua. https://www.in.gov.br/web/dou/-/portaria-n-122-de-23-de-marco-de-2021-310318921.

Brasil. Ministério da Saúde. Conselho Nacional de Saúde. (2012a). Resolução $N^{o} 466$, de 12 de dezembro de 2012: define diretrizes e normas regulamentadoras de pesquisas envolvendo seres humanos. http://bvsms.saude.gov.br/bvs/saudelegis/cns/2013/res0466_12_12_2012.html.

Brasil. Ministério da Saúde. Secretaria de Atenção à Saúde. (2012b). Manual sobre o cuidado à saúde junto a população em situação de rua. Ministério da Saúde.

Brasil. Ministério da Saúde. Secretaria de Vigilância em Saúde. Departamento de Vigilância das Doenças Transmissíveis. (2017a). Brasil Livre da Tuberculose: Plano Nacional pelo Fim da Tuberculose como Problema de Saúde Pública. Ministério da saúde.

Brasil. Portaria No 2.436, de 21 de setembro de 2017. (2017b). Aprova a Política Nacional de Atenção Básica, estabelecendo a revisão de diretrizes para a organização da Atenção Básica, no âmbito do Sistema Único de Saúde (SUS). https://bvsms.saude.gov.br/bvs/saudelegis/gm/2017/prt2436_22_09_2017.html.

Brasil. Ministério da Saúde. Secretaria de Vigilância em Saúde. (2019a). Guia de Vigilância em Saúde. Ministério da saúde.

Brasil. Ministério da Saúde. Secretaria de Vigilância Epidemiológica em Saúde, Departamento de Vigilância Epidemiológica. (2019b). Manual de recomendações para o controle da tuberculose no Brasil. ministério da Saúde.

Brasil. IBGE. População estimada: Campina Grande em 2020. (2020). Recuperado em 10 de outubro, 2020, de https://cidades.ibge.gov.br/brasil/pb/campinagrande/panorama.

Camargo, B. V. \& Justo, A. M. (2018). Tutorial para o uso do software Iramuteq: Interface de R pour les Analyses Multidimensionnelles de Textes et de Questionnaires. Florianópoles: Laboratório de Psicologia Social da Comunicação e Cognição. http://www.iramuteq.org/documentation/fichiers/tutoriel-enportugais.

Cunha, N. V., Cavalcanti, M. L. \& Costa, A. J. (2012). Diagnóstico situacional da descentralização do controle da tuberculose para a Estratégia Saúde da Família em jardim Catarina. Caderno de Saúde Coletiva, 20(2) 177-87. http://www.iesc.ufrj.br/cadernos/images/csc/2012_2/artigos/csc_v20n2_177-187.pdf.

Gil, A. C. (2017). Como elaborar projetos de pesquisa. Atlas.

Hino, P., Monroe, A. A., Takahashi, R. F., Souza, K. M. J., Figueiredo, T. M. R. M. \& Bertolozzi, M. R. (2018). Tuberculosis control from the perspective of health professionals working in street clinics. Revista Latino-Americana de Enfermagem, 26(3095) 1-9. http://www.scielo.br/scielo.php?script=sci_arttext\&pid=S010411692018000100397\&lng=en\&nrm=iso.

Riquelme D. J., Morales, V. J., Aguilera, V. R., Espinoza, O. M., Vidal, A. A. \& Riquelme, O. R. (2018). Impacto de la tuberculosis en el hospital de Puerto Montt. Revista Chilena de Enfermedades Respiratorias, 34 (3), 165-170. http://dx.doi.org/10.4067/s0717-73482018000300165.

Moreira, A. S. P. \& Jesuíno, J. C. (2003). Representações sociais: teoria e prática. Editora Universitária/UFPB.

Onwubiko, U., Wall, K., Sales, R. \& Holland, D. P. (2019). Using Directly Observed Therapy (DOT) for latent tuberculosis treatment - A hit or a miss? A propensity score analysis of treatment completion among 274 homeless adults in Fulton County, GA. Revista Plos One. 14(6). https://journals.plos.org/plosone/article?id=10.1371/journal.pone.0218373.

Queiroga, R. P. F., Sá, L. D. \& Grazzinelli, A. (2018). A tuberculose na população em situação de rua: desempenho de profissionais da atenção primária. Revista Rene (online), 19. https://pesquisa.bvsalud.org/portal/resource/pt/biblio-970824?lang=pt.7.

Santos, A. C. E., Brufentinker, C., Pena, L. S., Saraiva, S. S. \& Boing, A. F. (2021). Análise e comparação dos desfechos do tratamento de tuberculose na população em situação de rua e na população em geral do Brasil. Jornal Brasileiro de Pneumologia, 47(2) 1-4. http://www.jornaldepneumologia.com.br/details/3469/en-US/analysis-and-comparison-of-tuberculosis-treatment-outcomes-in-the-homeless-population-andin-the-general-population-of-brazil. 
Research, Society and Development, v. 10, n. 14, e24101421551, 2021

(CC BY 4.0) | ISSN 2525-3409 | DOI: http://dx.doi.org/10.33448/rsd-v10i14.21551

Santos, M. L. S. G., Vendramini, S. H. F., Gazetta, C. E., Oliveira, S. A. C. \& Villa, T. C. S. (2007). Pobreza: caracterização socioeconômica da tuberculose. Rev. Latino Americana de. Enfermagem, 15 (spe) 762-767. http://dx.doi.org/10.1590/S0104-11692007000700008.

Silva, T. O., Viana, P. J. S., Almeida, M. V. G., Santos, S. D. \& Nery, J. S. (2021). População em situação de rua no Brasil: estudo descritivo sobre o perfil sociodemográfico e da morbidade por tuberculose, 2014-2019. Revista Epidemiologia Serviço de Saúde, 30(1) 1-12. https://www.scielo.br/j/ress/a/PSxSGxP74bq473khC96GZmb/?lang=pt\#.

WHO. (2020). Global Tuberculosis Report 2020. Geneva: WHO. https://www.who.int/publications/i/item/9789240013131/.

Wysocki, A. D., Ponce, M. A. Z., Brunello, M. E F., Beraldo, A. A., Vendramini, S. H. F., Scatena, L. M., Netto, A. F. \& Villa, T. C. S. (2017). Primary Health Care and tuberculosis: services evaluation. Revista Brasileira de Epidemiologia 20(1) 161-75, 2017. http://dx.doi.org/10.1590/19805497201700010014.

Zuim, R. C. B. \& Trajman, A. (2018). Itinerário terapêutico de doentes com tuberculose vivendo em situação de rua no Rio de Janeiro. Revista Physis, 20(2). http://www.scielo.br/scielo.php?script=sci_arttext\&pid=S0103-73312018000200601\&lng=pt\&nrm=iso. 